\title{
Os Caminhos da Modelagem no "Pós”-GPIMEM: desenvolvendo uma árvore genealógica
}

\author{
The Routes of Modelling in "After-GPIMEM": developing a family tree
}

\author{
Débora da Silva Soares ${ }^{1}$ \\ debora.soares@ufrgs.br
}

Marcelo C. Borba ${ }^{2}$

gpimem@rc.unesp.br

\begin{abstract}
Resumo
Neste artigo analisamos os caminhos seguidos por professores/pesquisadores, ex-alunos do Grupo de Pesquisa em Informática outras Mídias e Educação Matemática (GPIMEM), no desenvolvimento de pesquisas sobre modelagem matemática enquanto estratégia pedagógica. Nesse sentido, procuramos identificar que questões foram tematizadas em suas pesquisas sobre o tema. Elaboramos uma árvore genealógica desses trabalhos, tomando como ponto de partida aqueles apresentados em Malheiros e Franchi (2013), os quais se referem às pesquisas de mestrado e/ou doutorado, desenvolvidas por esses autores, enquanto estudantes do grupo. Expandimos a árvore genealógica considerando as pesquisas desenvolvidas por esses mesmos autores, porém enquanto professores/pesquisadores/orientadores em universidades do país e do exterior. Nossa análise indicou uma continuidade na variedade de temas abordados pelas pesquisas, assim como a presença de tecnologias nesses estudos. Nesse último caso, identificamos alguns papéis possíveis para as tecnologias no desenvolvimento de projetos de modelagem.
\end{abstract}

Palavras-chave: Educação Matemática. Modelagem Matemática. Tecnologias.

\begin{abstract}
In this paper we analyze the paths followed by professors/researchers, former students of the research group GPIMEM, in the development of research about mathematical modeling as a pedagogical approach. We sought to identify the questions discussed by these researchers in their studies. We developed a family tree of these studies, using as a starting point those presented in Malheiros and Franchi (2013), which refers to masters and/or doctoral research developed by the authors included in the analysis. We expanded this family tree considering the studies developed by these same authors as professors/researchers/advisors in universities of Brazil and

${ }^{1}$ Doutora em Educação Matemática pela Universidade Estadual Paulista (UNESP), Rio Claro, SP, Brasil. Professora do Programa de Pós-Graduação em Ensino de Matemática, Departamento de Matemática Pura e Aplicada, da Universidade Federal do Rio Grande do Sul (UFRGS), Porto Alegre, RS, Brasil. Membro Associado do GPIMEM. Endereço para correspondência: Av. Bento Gonçalves, 9500, Prédio 43-111, Agronomia, CEP: 91509-900, Porto Alegre, RS, Brasil.

2 Livre Docente pela Universidade Estadual Paulista (UNESP). Professor do Programa de Pós-Graduação em Educação Matemática da Universidade Estadual Paulista (UNESP) e coordenador do Grupo de Pesquisa em Informática outras Mídias e Educação Matemática (GPIMEM), Rio Claro, SP, Brasil. Endereço para correspondência: Departamento de Matemática - UNESP. Avenida 24A, 1515, Bela Vista, CEP: 13506-900, Rio Claro, SP, Brasil.
\end{abstract}


abroad. Our analysis suggests continuity in the diversity of themes addressed by the studies, as well as the presence of technologies. In this last case, we identified some possible roles of technology in the development of modeling projects.

Keywords: Mathematics Education. Mathematical Modelling. Technologies.

\section{Introdução}

Segundo o dicionário Houaiss (2001, p.1440), genealogia refere-se ao "estudo que tem por objeto estabelecer a origem de um indivíduo ou de uma família". Além disso, a evolução e a disseminação dessa família também são focos de estudo dessa ciência e, frequentemente, árvores genealógicas são elaboradas para ajudar nesse fim. Uma árvore genealógica é uma representação gráfica que apresenta os indivíduos da família evidenciando sua relação de parentesco (traços horizontais indicam uma relação matrimonial, e traços verticais indicam uma relação parental/filial).

Nesse artigo, vamos modificar um pouco o contexto de origem de uma árvore genealógica e vamos utilizá-la como um instrumento para analisar os desdobramentos que a pesquisa sobre modelagem matemática enquanto estratégia pedagógica tomou no Grupo de Pesquisa em Informática outras Mídias e Educação Matemática (GPIMEM) ao longo dos seus 20 anos de existência. Para isso, tomaremos como ponto de partida o trabalho de Malheiros e Franchi (2013), cujos objetivos foram: apresentar o papel das tecnologias nas produções sobre modelagem desenvolvidas no grupo e o modo como as pesquisas tematizam as possibilidades de entrelaçamento entre elas; refletir sobre como "novas" e "velhas" tecnologias podem coexistir com "novas" e "velhas" práticas de modelagem. Na verdade, esse trabalho, embora seja ponto de partida, pode também ser considerado "como ponto de chegada", já que ele sintetiza vinte anos do trabalho de um grupo de pesquisa em uma dada linha de pesquisa.

A análise realizada por Malheiros e Franchi (2013) tomou como base os trabalhos envolvendo modelagem e tecnologias desenvolvidos no contexto de pesquisas de mestrado e doutorado. Atualmente, a maioria dos autores desses trabalhos atua como professores e pesquisadores de diferentes universidades do país e do exterior, de modo que nos pareceu interessante expandir a análise iniciada pelas autoras supracitadas e tomar como base agora os trabalhos desenvolvidos por esses autores sobre modelagem enquanto professores, orientadores e pesquisadores em suas universidades. 
Nesse sentido, o objetivo do presente artigo é identificar que questões sobre modelagem são tematizadas em pesquisas desenvolvidas por ex-alunos de mestrado e/ou doutorado do GPIMEM enquanto professores, orientadores e pesquisadores em suas universidades, de modo a analisar os caminhos da modelagem seguidos por esses autores. A partir do material analisado, elaboramos uma árvore genealógica do grupo tendo como foco as pesquisas em modelagem.

Para isso, iniciaremos apresentando um breve resumo da análise feita por Malheiros e Franchi (2013), elaborando um primeiro estágio da árvore genealógica. $\mathrm{Na}$ sequência, acrescentaremos a essa árvore os trabalhos de modelagem não contemplados por essas autoras. Finalmente, apresentaremos os trabalhos orientados ou realizados por cada pesquisador, identificando as questões tematizadas e ampliando a árvore genealógica. Esperamos obter um instrumento visual a partir do qual será possível analisar: as "origens" dos trabalhos em termos de orientação, os principais questionamentos abordados sobre o tema modelagem e a disseminação dos membros do grupo pelo país e exterior.

Em termos metodológicos, a estruturação dessas árvores genealógicas foi desenvolvida com base na análise de publicações feitas pelos pesquisadores. A primeira árvore, como mencionado acima, foi elaborada com base no texto de Malheiros e Franchi (2013). A segunda árvore é uma complementação da primeira, sendo que seus dados partiram do acervo de teses e dissertações do grupo. Já a terceira árvore foi elaborada, em um primeiro momento, com base em informações obtidas com os próprios autores por meio de emails e/ou por meio de consulta a seus currículos Lattes. Em um segundo momento, os trabalhos publicados relevantes para nosso tema de investigação foram estudados para a identificação das questões tematizadas.

\section{Modelagem como foco de pesquisa de mestrados e doutorados no GPIMEM}

Vamos iniciar a elaboração de nossa árvore genealógica considerando os estudos sobre modelagem desenvolvidos no GPIMEM enquanto pesquisas de mestrado e doutorado. Conforme apontam Diniz e Borba (2012) com base na análise de anais dos principais eventos do país, o grupo é responsável por boa parte das pesquisas que envolvem modelagem, tecnologias e suas relações. Esse fato se reflete nas dissertações e teses desenvolvidas no grupo que, em sua maioria, tratam de modelagem e tecnologias. 
Como mencionamos na introdução, Malheiros e Franchi (2013) analisaram o papel das tecnologias na produção sobre modelagem nos trabalhos desenvolvidos por membros do GPIMEM enquanto alunos de mestrado e/ou doutorado. As autoras iniciam situando o leitor historicamente, informando que a inserção da modelagem enquanto tema de pesquisa no grupo se deu pela influência de seu coordenador, professor Marcelo de Carvalho Borba, cuja dissertação de mestrado envolveu o trabalho com modelagem com crianças de uma favela da periferia de Campinas. Mais tarde, os trabalhos de modelagem foram (e ainda são) propostos aos alunos do curso de Ciências Biológicas da Unesp, Rio Claro, SP, na disciplina Matemática Aplicada. A concepção de modelagem adotada por Borba é a de que os alunos, em pequenos grupos, escolhem um tema de seu interesse para pesquisar e o professor trabalha como um orientador nas questões que envolvem o estudo do tema com auxílio da matemática.

Na sequência, as autoras apresentam as pesquisas de mestrado e doutorado desenvolvidas no grupo sobre modelagem, e analisam os resultados de cada uma com relação às tecnologias e o seu papel. Malheiros e Franchi (2013) relatam a forma natural com que as diferentes tecnologias foram sendo incorporadas aos trabalhos de modelagem ao longo dos anos. As autoras identificam vários papéis para as tecnologias no fazer modelagem:

Podemos dizer que hoje elas são utilizadas para investigar, coletar dados, problematizar, comparar, visualizar, interpretar e também para comunicação ao longo das diferentes etapas das diversas atividades de Modelagem desenvolvidas pelo Grupo. A experimentação e simulação proporcionadas pelas TIC, a exploração de conteúdos matemáticos, o uso de softwares para traçar gráficos, dentre outras possibilidades, parecem estar incorporadas à Modelagem em suas diferentes facetas (MALHEIROS; FRANCHI, 2013, p.187).

Esses diferentes papéis das tecnologias identificados pelas autoras exemplificam a sinergia (BORBA; PENTEADO, 2001) que pode existir entre as tecnologias e a modelagem, isto é, ilustram as possibilidades que diferentes mídias podem oferecer para um trabalho investigativo e aberto como é aquele proposto pela modelagem. Nesse sentido, a visão epistemológica sobre as tecnologias que perpassa os trabalhos do grupo, baseada no construto teórico seres-humanos-com-mídias (BORBA; VILLARREAL, 2005), enfatiza que as tecnologias possuem um papel central na produção de conhecimento, na medida em que reorganizam o pensamento humano, assim como o moldam e são por ele moldadas. Além disso, a unidade de produção de conhecimento é entendida como um coletivo formado por 
humanos e pelas mídias com as quais eles trabalham (sejam computadores, calculadoras e/ou lápis e papel).

Esse resumo, apesar de breve, aponta os principais elementos que servirão de ponto de partida para o desenvolvimento desse trabalho. A seguir (Fig.1), apresentamos a primeira parte da árvore genealógica que desenvolvemos a partir do estudo de Malheiros e Franchi (2013). Tomamos como indivíduos as pesquisas de mestrado e doutorado desenvolvidas no grupo sobre modelagem e tecnologias e seus respectivos autores ${ }^{3}$. Para cada um deles apresentamos os principais resultados com relação ao papel das tecnologias no fazer modelagem. Observamos que todas as pesquisas adotaram a modelagem matemática (em diferentes concepções) enquanto estratégia pedagógica.

Em nossa representação, os traços diagonais, que ligam um nível a outro, indicam uma relação de orientação; já os traços horizontais ligam trabalhos de mestrado e doutorado de um mesmo autor. O trabalho de Franchi (2002) aparece desconectado dos demais, pois, apesar de sua autora não ter feito nem o mestrado e nem o doutorado como orientanda no GPIMEM, ela atuou enquanto doutora no grupo, o qual influenciou seu trabalho de professora e pesquisadora.

Uma análise dessa árvore genealógica nos permite observar uma evolução nos resultados obtidos com relação ao papel das tecnologias no fazer modelagem. Mesmo com diferentes contextos e com diferentes questões de pesquisa, o papel da tecnologia foi observado em cada um desses trabalhos, uma vez que, como entendemos e mencionamos anteriormente, a tecnologia possui um papel central no processo de produção de conhecimento. Conforme novas tecnologias foram sendo incorporadas, outros papéis foram sendo observados, como em Diniz (2007) e em Malheiros (2008) com relação à internet. É possível ver que os trabalhos formam um mosaico de pesquisas, na medida em que os resultados de cada pesquisa se complementam e enriquecem a compreensão sobre o papel das mídias no fazer modelagem.

\footnotetext{
${ }^{3}$ Todos os autores e resultados aqui citados também foram citados por Malheiros e Franchi (2013), com exceção de Vechia (2012) e Soares (2012), dos quais as autoras citam outros trabalhos já que suas pesquisas ainda não estavam concluídas na ocasião de escrita do artigo. Sugerimos a leitura desse trabalho para que se tenha conhecimento dos diferentes contextos e questões de investigação de cada pesquisa.
} 


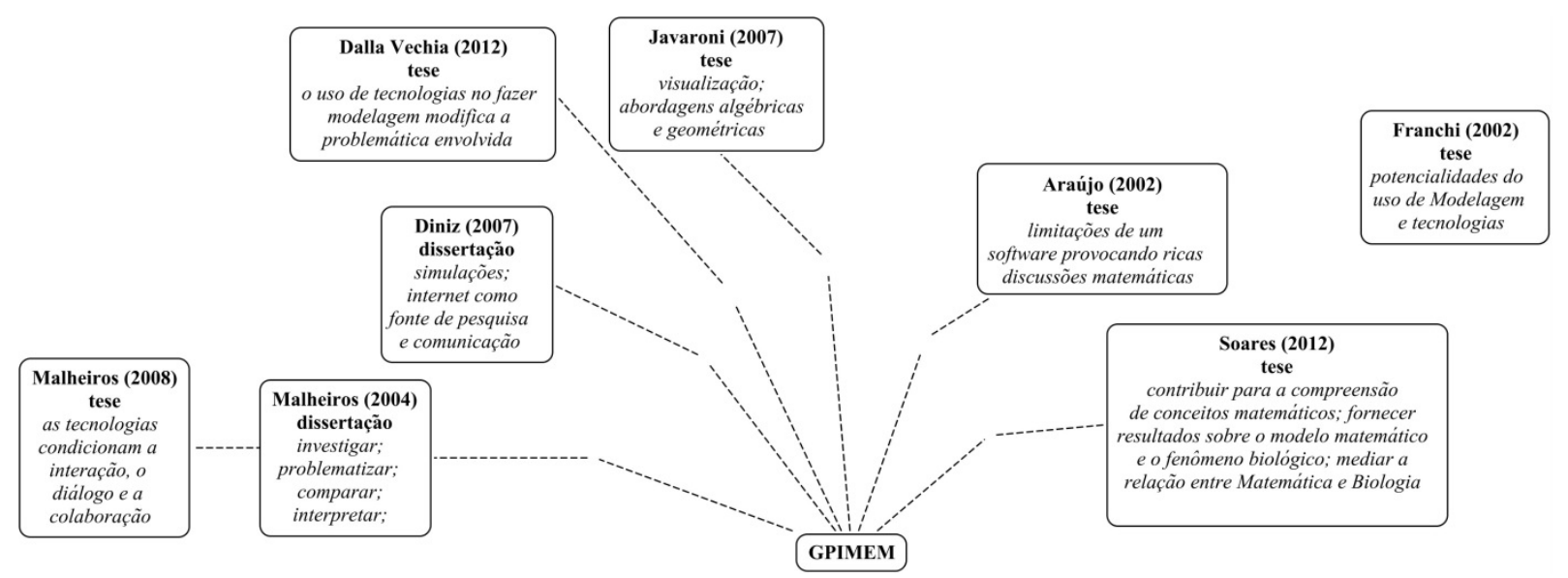

Figura 1: Árvore genealógica sobre os papéis das tecnologias nas pesquisas de mestrado e/ou doutorado sobre modelagem desenvolvidas no GPIMEM.

Ainda com base nesses mesmos autores, elaboramos uma segunda árvore genealógica (Fig.2), a qual apresenta, de forma resumida, o foco de cada uma das pesquisas supracitadas. Acrescentamos a ela outros dois trabalhos que enfocam a modelagem, desenvolvidos no grupo como pesquisas de mestrado e doutorado, e que não estiveram no levantamento anterior, uma vez que suas pesquisas não tratavam do uso de tecnologias. São eles: Herminio (2009) e Barbosa (2001).

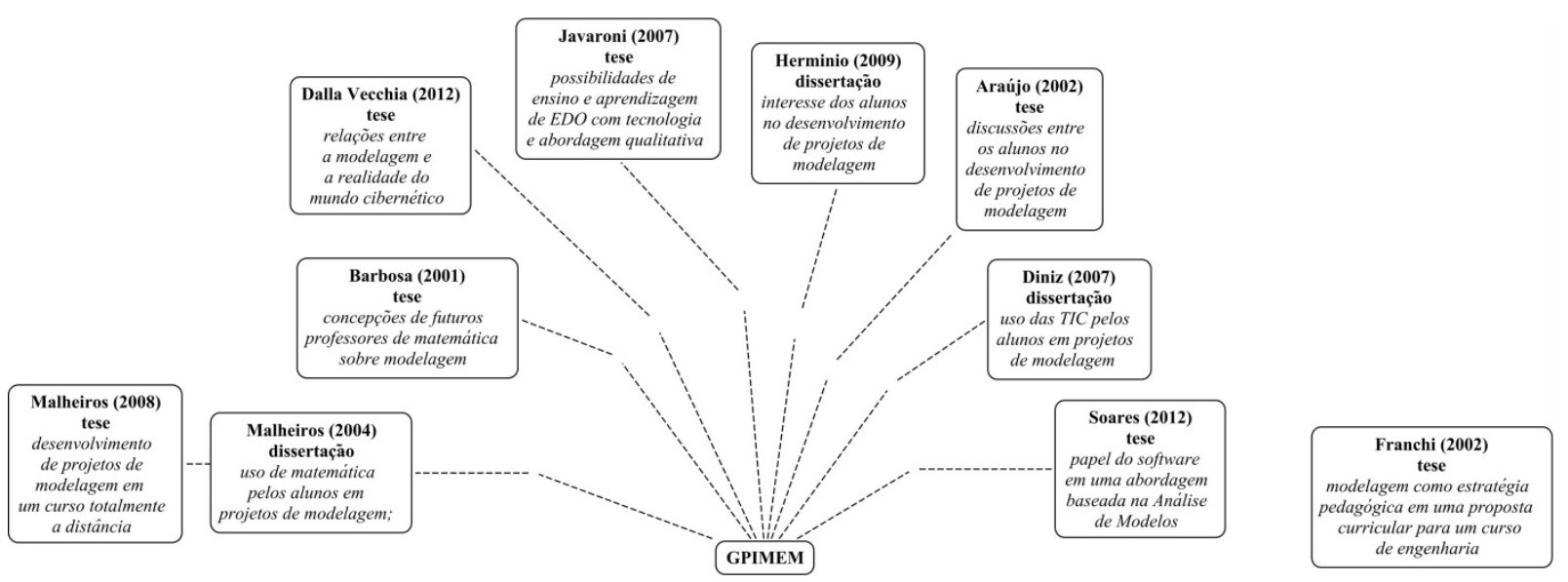

Figura 2: Árvore genealógica sobre as questões investigadas em pesquisas de mestrado e/ou doutorado sobre modelagem no GPIMEM.

Uma análise dessa árvore genealógica (Fig.2) nos permite identificar o trabalho de Herminio (2009), que enfocou um tema ainda pouco questionado na literatura de modelagem, que é o 
interesse dos alunos na escolha dos temas para investigação. A pesquisa indicou a complexidade envolvida nesse processo, discutindo questões como a perda de interesse ou a escolha do tema pelos alunos tendo como intenção agradar ao professor. Já o trabalho de Barbosa (2001) investigou as concepções de futuros professores de Matemática sobre modelagem quando eles têm contato com esse ambiente de aprendizagem, destacando a influência das experiências matemáticas na formação dessas concepções, assim como de elementos externos ao professor e do seu saber-fazer. Também apresentou a concepção sociocrítica de modelagem, destacando a matemática como um meio para compreensão de temas do cotidiano ou de outras disciplinas.

Além disso, também é possível verificar a diversidade de temas relacionados à modelagem abordados pelas pesquisas de mestrado e/ou doutorado desenvolvidas no grupo. Novamente é possível notar que, mesmo questionamentos não específicos sobre a tecnologia levaram à discussão sobre seu uso no desenvolvimento de projetos de modelagem. O que nos questionamos é se essa variedade de temas sobre a modelagem se propagou para as pesquisas dos ex-alunos do grupo. Em outras palavras, que caminhos a modelagem tomou nas pesquisas desenvolvidas por esses autores enquanto professores, pesquisadores e orientadores.

\section{Modelagem enquanto foco de pesquisa de professores, orientadores e pesquisadores formados no GPIMEM}

Nessa seção, nosso intuito é abordar as pesquisas sobre modelagem desenvolvidas pelos exalunos do GPIMEM enquanto professores, pesquisadores e orientadores em suas universidades, expandindo a árvore genealógica apresentada na Fig.2. Para isso, faremos uma pequena apresentação de cada autor, de modo a contextualizar sua atuação e, em seguida, trataremos das pesquisas sobre modelagem realizadas enquanto pesquisador e/ou orientador, observando que outras questões relativas à modelagem chamaram a atenção dos autores.

A seção está organizada em subseções, cujos títulos são as regiões do país em que os professores/pesquisadores estão atualmente alocados. Dessa forma também será possível mapear a disseminação geográfica desses autores, formados no GPIMEM e que mantêm seu interesse pela modelagem.

Região Sudeste 
Iniciamos apresentando a $\operatorname{Prof}^{\mathrm{a}}$. Dr ${ }^{\mathrm{a}}$. Sueli Javaroni, que atualmente é professora do Departamento de Matemática da Unesp, campus de Bauru, e é pesquisadora/orientadora do GPIMEM. O doutorado de Javaroni versou sobre o ensino de equações diferenciais ordinárias com o uso de tecnologias (JAVARONI, 2007). Seu foco de estudo foi analisar quais as possibilidades de ensino e aprendizagem de equações diferenciais ordinárias (sua introdução) a partir de uma abordagem qualitativa de modelos matemáticos com o uso de tecnologias, em particular, os softwares Winplot e Excel. Em sua tese, a autora desenvolveu algumas atividades, às quais trabalhou com alguns alunos em horário extraclasse.

Logo após seu doutorado, Javaroni submeteu um projeto trienal, o qual teve como foco a reformulação das atividades propostas no doutorado. Durante esse projeto, Javaroni orientou três iniciações científicas, que estiveram vinculadas a ele. Uma delas esteve relacionada a um projeto do Núcleo, desenvolvido em parceria com a Prof ${ }^{a}$. Dr ${ }^{a}$. Ivete Baraldi, o qual envolveu narrativas, modelagem matemática e tecnologias. O principal objetivo do projeto foi “fomentar discussões e reflexões acerca da utilização de Narrativas e Modelagem Matemática, com o auxílio de tecnologias informáticas para o desenvolvimento de projetos de ensino e aprendizagem de conteúdos de matemática" (COELHO et al., 2010), o qual se preocupou tanto com a formação inicial quanto continuada de professores de matemática.

Nessa pesquisa, propôs-se um tema gerador - o valor nutricional dos alimentos - como ponto de partida do trabalho com professores de matemática, que foram convidados a elaborar narrativas para registrar memórias de suas práticas docentes, assim como debatê-las e refletir sobre as mesmas. Na sequência, desenvolveram-se projetos de modelagem relacionados ao tema gerador com o uso de softwares usualmente encontrados nos computadores das escolas (como o Excel, por exemplo).

Um segundo trabalho de iniciação científica, orientado por Javaroni, teve como objetivo o desenvolvimento de um applet para a exploração de alguns dos modelos matemáticos utilizados pela autora no desenvolvimento de sua tese de doutorado. O applet desenvolvido pode ser compreendido como um "interpretador" das equações diferenciais ordinárias, uma vez que ele as analisa e elabora uma representação do seu campo de direções (conjunto de vetores diretores da equação) (SANTOS; JAVARONI, 2012). Segundo Santos e Javaroni (2012) o applet possibilita novas formas de exploração das equações diferenciais ordinárias e as informações visuais e resultados numéricos fornecidos pelo software podem contribuir para 
que o estudante compreenda melhor de que forma o fenômeno, representado por uma determinada equação, evolui.

A terceira orientação de iniciação científica teve como objetivo o desenvolvimento de atividades investigativas sobre alguns modelos matemáticos envolvendo conteúdos do cálculo numérico computacional e que utilizaram softwares geométricos e algébricos. Alguns dos softwares utilizados foram: MatLab, Microsoft Excel e Dev C++. (SGARIBOLDI; JAVARONI, 2013). Para cada situação escolhida, estudou-se um modelo matemático relacionado, assim como métodos numéricos para sua resolução, os quais foram desenvolvidos com os diferentes softwares trabalhados. Segundo Sgariboldi e Javaroni (2013), foi possível desenvolver uma maneira acessível para o leitor formular um modelo matemático e solucioná-lo por meio de métodos numéricos.

Ainda no estado de São Paulo, a Prof ${ }^{\mathrm{a}}$. Dr ${ }^{\mathrm{a}}$. Ana Paula dos Santos Malheiros atua na Graduação na Universidade Estadual Paulista (Unesp), campus de Rio Preto, SP, e na PósGraduação em Educação Matemática da mesma universidade, campus de Rio Claro, SP. Participa do GPIMEM como pesquisadora e orientadora, e desenvolveu suas pesquisas de mestrado e doutorado no grupo. Seu interesse no mestrado foi investigar como os alunos utilizam o conteúdo de matemática no desenvolvimento de projetos de modelagem (MALHEIROS, 2004). Já no doutorado, sua pesquisa tratou de como projetos de modelagem são desenvolvidos em um curso totalmente a distância (MALHEIROS, 2008).

Atualmente, seu principal foco de pesquisa é compreender como a modelagem, baseada na filosofia de educação de Paulo Freire, pode contribuir na formação inicial de professores de Matemática. Dentro desse escopo, em Malheiros (2013), a autora apresenta possíveis diálogos entre a modelagem e as ideias de Paulo Freire. A concepção de modelagem adotada pela autora a considera uma abordagem pedagógica na qual os estudantes escolhem um tema de seu interesse para investigar e utilizam a matemática para conduzir essa investigação, sempre orientados pelo professor. Nessa concepção, "a criticidade, o diálogo, a problematização e a autonomia são fundamentais" (MALHEIROS, 2013, p.4). Todos esses aspectos são defendidos por Paulo Freire como essenciais para o desenvolvimento de uma educação libertadora.

Segundo Malheiros (2013), Paulo Freire sugere o desenvolvimento do ensino tendo como base as Investigações Temáticas, isto é, a proposição de temas de interesse dos estudantes 
para que sejam investigados, debatidos e refletidos, de modo que seja desenvolvida uma compreensão crítica do mesmo. A autora argumenta que a modelagem pode ser desenvolvida de forma bastante próxima das ideias de Paulo Freire e, portanto, se tornar uma alternativa para o desenvolvimento da filosofia de Freire no ensino de matemática nos diferentes níveis escolares.

No estado de Minas Gerais, a Prof ${ }^{a}$. Dr ${ }^{\mathrm{a}}$. Regina Franchi trabalha na Universidade federal de Outro Preto, atuando na Graduação e na Pós-Graduação, mais especificamente no Mestrado Profissional em Educação Matemática. Sua tese foi defendida em 2002 e teve o professor Marcelo Borba como participante de sua banca. Franchi vinculou-se ao GPIMEM após a defesa de seu doutorado e participou mais ativamente do grupo a partir de 2007. Seu foco de interesse no doutorado foi a modelagem matemática como estratégia pedagógica em uma proposta curricular para cursos de engenharia. Franchi é membro associada ${ }^{4}$ do GPIMEM.

Em sua atuação na Pós-Graduação, Franchi possui orientações de mestrado sobre diferentes temas. Em particular, possui uma orientação de mestrado concluída, cujo objetivo foi investigar quais as contribuições que uma proposta baseada na modelagem, no uso de tecnologias e na perspectiva da Educação Matemática Crítica pode trazer para a abordagem do conceito de função (FRANCHI; FERREIRA, 2013). A pesquisa foi desenvolvida com estudantes do Ensino Médio, que escolheram temas de seu interesse para pesquisar. Com base nos dados coletados pelos alunos para o desenvolvimento de suas pesquisas, foram elaboradas atividades com o intuito de debater o conceito de função. As discussões enfatizaram os momentos em que a perspectiva sociocrítica da modelagem pôde ser evidenciada, o que ocorreu principalmente nas discussões coletivas sobre os assuntos investigados pelos alunos.

Também em Minas Gerais, a Prof ${ }^{\mathrm{a}}$. Dr ${ }^{\mathrm{a}}$. Jussara de Loiola Araújo atua na Universidade Federal de Minas Gerais (UFMG) tanto na Graduação como na Pós-Graduação, especificamente no Programa de Pós-Graduação em Educação. Ela participa do GPIMEM, como membro associada, e é coordenadora de outros dois grupos de pesquisa: o Grupo de Estudos e Pesquisa em Educação Matemática, Modelagem e Tecnologias e o Grupo de Pesquisa e Estudos Histórico-Culturais em Educação Matemática e em Ciências. Sua tese de

\footnotetext{
${ }^{4}$ O GPIMEM é composto por membros plenos, ou seja, aqueles que em geral vivem o cotidiano presencial e virtual do grupo e tem seu vínculo principal com este grupo. Há também os membros associados, que em geral se vincularam ou lideram outros grupos, mas tem ao menos uma parceria na pesquisa que desenvolvem com os membros plenos. Em outro tipo de recorte o grupo se divide entre os membros nacionais e de outros de países como a Argentina e Colômbia.
} 
doutorado (ARAÚJO, 2002) teve como foco as discussões desenvolvidas em um ambiente de ensino e aprendizagem de Cálculo envolvendo modelagem e o uso de tecnologias informáticas.

Enquanto pesquisadora e orientadora do Programa de Pós-Graduação em Educação da UFMG, diferentes temas perpassaram a pesquisa de Araújo e de seus orientandos. Com relação à modelagem, há duas temáticas em pesquisas de mestrado já concluídas: a modelagem matemática e a experiência do professor; e a modelagem matemática e o envolvimento dos alunos. Em nível de doutorado, uma das pesquisas, também já concluída, investigou como ocorre o processo de matematização no desenvolvimento de um projeto de modelagem orientado pela perspectiva da Educação Matemática Crítica. Um dos apontamentos da pesquisa é que o processo de matematização desenvolveu-se em parceria entre o grupo de alunos e tecnologias informáticas que, em alguns casos, estavam presentes no trabalho de disciplinas técnicas do curso. Segundo Freitas (2011) o uso de tecnologias permitiu um maior dinamismo às investigações desenvolvidas no processo de modelagem e, juntamente com a abordagem sociocrítica da modelagem, constituiu-se como uma possibilidade para desafiar a Ideologia da Certeza (BORBA; SKOVSMOSE, 2001).

Região Sul

O Prof. Dr. Rogrigo Dalla Vecchia é coordenador do curso de Matemática e professor colaborador do Programa de Pós-Graduação em Ensino de Ciências e Matemática da Universidade Luterana do Brasil (Ulbra), Canoas, RS. Ele divide a coordenação do Grupo de Pesquisa em Ambientes Matemáticos de Aprendizagem com a Inclusão da Informática na Sociedade (AMAIIS) com o Prof. Dr. Maurício Rosa. Em sua pesquisa de doutorado, o principal interesse foi em compreender as relações entre a Modelagem Matemática e a realidade do mundo cibernético (DALLA VECCHIA, 2012).

Após a finalização de sua pesquisa de doutorado, Dalla Vecchia iniciou um estudo em que procura aprofundar o entendimento sobre uma das problemáticas abordadas em seu doutoramento, a saber, a gênese e a transformação da determinação do problema associado ao processo de modelagem matemática. Essa questão de pesquisa está sendo desenvolvida juntamente com uma orientação de mestrado, a qual lida com tópicos de Pesquisa Operacional para modelar e resolver problemas emergentes do trabalho de estudantes de Engenharia de Produção. Os trabalhos de Deleuze (1988) são fonte fundamental para a discussão que está 
sendo elaborada pelos autores, em particular no aspecto relacionado à visão de problema e em sua importância/influência no processo de modelagem.

O artigo de Dalla Vecchia e Maltempi (2012) apresenta algumas ideias a respeito do tema supracitado. Os autores justificam a necessidade de um aprofundamento sobre o conceito de problema devido ao fato de que a modelagem, "de modo direto ou indireto, parece estar associada a uma situação problemática (DALLA VECCHIA; MALTEMPI, 2012, p.203). Baseando-se nos trabalhos de Deleuze (1988), os autores entendem que um problema não está diretamente associado à proposição que o representa, mas é anterior a ela. Além disso, a própria proposição de certa forma já indica possíveis respostas e caminhos pelos quais o problema pode se desenvolver.

Esse entendimento caracteriza um problema como sendo dinâmico e mutável o que, por sua vez, sustenta a visão de modelagem dos autores como um processo em transformação. Segundo Dalla Vecchia e Maltempi (2012), a dinamicidade do problema não está apenas em buscar diferentes representações para o mesmo (por exemplo, por meio da Matemática), mas também no modo como ele é analisado e compreendido. Nesse sentido, a tecnologia pode ser um vetor de mudança, propiciando maneiras diferentes de analisar determinado problema e possibilitando diferentes formas de compreendê-lo.

Também no Rio Grande do Sul, a Prof ${ }^{\mathrm{a}}$. Dra . Débora da Silva Soares é professora do Programa de Pós-Graduação em Ensino de Matemática da Universidade Federal do Rio Grande do Sul (UFRGS). Sua atuação na Graduação ocorre principalmente no curso de Licenciatura em Matemática. Em sua pesquisa de doutorado (SOARES, 2012) o foco central foi analisar qual o papel de um software no desenvolvimento de uma abordagem pedagógica para trabalhar conceitos de Cálculo Diferencial com alunos de Biologia. Essa abordagem teve como base a análise de um modelo matemático para um fenômeno biológico (a transmissão da malária), modelo que envolve um sistema de duas equações diferenciais ordinárias não lineares.

As discussões iniciadas nessa pesquisa incentivaram a autora a aprofundar a compreensão sobre a produção dos estudantes durante o trabalho com a proposta elaborada. Nesse sentido, um novo projeto de pesquisa está se delineando, cujo objetivo será analisar as contradições internas (ENGESTRÖM; SANNINO, 2010) enfrentadas pelos estudantes ao discutirem 
conceitos do Cálculo Diferencial em um ambiente com tecnologia e tendo como base um fenômeno de outra área científica, assim como um modelo matemático para o mesmo.

\section{Região Nordeste}

O Prof. Ms. Leandro Diniz é docente da Universidade Federal do Recôncavo da Bahia (UFRB) e trabalha com a Licenciatura em Matemática, ministrando uma disciplina que trata sobre modelagem e outra sobre tecnologias. Nessas disciplinas, Diniz propõe a realização de projetos de modelagem pelos alunos. Dois relatos de experiências tratando do desenvolvimento desses projetos foram publicados com os licenciandos, um no Encontro Nacional de Educação Matemática (ENEM) em 2010 e o outro no Encontro Baiano de Educação Matemática (EBEM) em 2011.

Diniz participa do Grupo de Pesquisa Educação Matemática no Recôncavo da Bahia (GPEMAR), que contempla todos os docentes da área de Educação Matemática da Universidade. Conforme ele descreve, o grupo funciona como uma espécie de "guardachuva", que acolhe a todos, assim como aos subgrupos formados de acordo com áreas mais específicas de pesquisa. Da mesma forma que outros docentes da universidade, Diniz está cursando o doutorado em Educação na Universidade do Minho, num convênio internacional com a UFRB, na linha de pesquisa em Educação Matemática. Diniz investiga, em sua tese, a construção, leitura e interpretação de gráficos estatísticos em projetos de modelagem com uso das tecnologias digitais, a partir do estudo da complexidade semiótica dos gráficos estatísticos utilizados por alunos de um curso de nível médio técnico. A pesquisa está em fase inicial, mas as primeiras ideias foram discutidas em Diniz e Borba (2012), que focam nos dados prontos coletados na internet. Em Diniz e Souza (2013), há reflexões sobre que aspectos considerar na elaboração de questões sobre interpretação de gráficos estatísticos.

Alguns dos trabalhos de conclusão de curso orientados por Diniz têm a modelagem como foco de interesse. Por exemplo, Andrade (2010) analisou as contribuições da modelagem para a vida cotidiana de alunos da Educação de Jovens e Adultos (EJA). A partir da observação do interesse dos alunos por questões habitacionais, Andrade propôs o desenvolvimento de um projeto de modelagem cuja problemática era a construção de uma casa ideal para uma família carente. Informações sobre a construção de casas e sobre a escala para a elaboração de plantas foram pesquisadas na internet. Em grupos, os alunos elaboraram propostas, que foram avaliadas por pedreiros no momento de sua apresentação oral. A partir dessa avaliação, 
elegeu-se uma das propostas, a qual foi entregue à prefeitura da cidade. A autora observou que a modelagem pode proporcionar o desenvolvimento de atitudes positivas nos alunos com relação à matemática, contribuir para valorizar a pluralidade sociocultural e criar condições para que alunos possam ser agentes de transformação de seu contexto social.

Souza (2013) investigou as contribuições que a modelagem pode proporcionar para que alunos do $7^{\circ}$ ano do Ensino Fundamental atribuam significados aos conteúdos de matemática. O projeto de modelagem foi desenvolvido a partir de um interesse dos alunos: a construção de uma proteção de tela na quadra da escola. A análise dos dados revelou que as discussões e o comportamento dos alunos desenrolaram-se de modo ativo no processo de ensino e aprendizagem da matemática, produzindo significados para os conteúdos matemáticos (por exemplo, a associação entre o conceito de perímetro e o comprimento da cerca).

Também na Bahia, o Prof. Dr. Jonei Barbosa é docente da Universidade Federal da Bahia (UFBA). Ele trabalha com a Licenciatura em Matemática e no Programa de Pós-Graduação em Educação e em Ensino, Filosofia e História das Ciências (PPGEFHC). Já ministrou disciplinas na PPGEFHC sobre modelagem e tendências em educação matemática e orientou dissertações e teses sobre modelagem, com temáticas envolvendo EJA, formação de professores, dentre outros. Mais recentemente, passou a desenvolver pesquisas com foco nos estudos do sociólogo Basil Bernstein. Atualmente, Barbosa não foca sua pesquisa em modelagem, pois se dedica a analisar a mudança da prática pedagógica de professores de matemática, a partir de formação (em cursos) com o uso de materiais curriculares educativos (SILVA et al., 2013).

Dentre as pesquisas orientadas por Barbosa, destacamos as questões investigadas por duas das três teses de doutorado relacionadas ao tema de modelagem. Oliveira (2010) investigou as tensões nos discursos de professores quando do trabalho com a modelagem em sala de aula, onde o conceito de tensões refere-se a descontinuidades entre o discurso produzido na prática de sala de aula e o discurso sobre modelagem. Já a pesquisa de Souza (2012) teve como foco de estudo que aprendizagem matemática se constitui no fazer modelagem matemática. Ela sugere que a aprendizagem pode ser entendida como "a aprendizagem da maneira de usar as palavras à luz da matemática escolar e da identificação de semelhanças entre esses usos e os usos das palavras sugeridos pelas situações-problema" (SOUZA, 2012, p.9, grifos do autor).

\section{Argentina}


A Prof ${ }^{a}$. Dra . Mónica Ester Villarreal é docente da Facultad de Matemática, Astronomía e Física da Universidad Nacional de Córdoba (UNC), Córdoba, Argentina. Ela atua como pesquisadora do Consejo Nacional de Investigaciones Científicas e Técnicas (CONICET) e é membro do grupo de pesquisa Grupo de Enseñanza de la Ciencia y la Tecnología (GECyT), o qual reúne pesquisadores de Educação Matemática e de Ensino de Física. Sua área de interesse é a prática docente em cenários de modelagem.

Enquanto doutoranda do GPIMEM, a Prof ${ }^{a}$. Mónica desenvolveu sua pesquisa com foco nas tecnologias, em particular sobre os processos de pensamento matemático de estudantes de Cálculo Diferencial e Integral ao trabalharem questões relacionadas ao conceito de derivada em um ambiente computacional (VILLARREAL, 1999). Já enquanto professora e pesquisadora da Universidade Nacional de Córdoba, seu interesse se voltou para a modelagem e, portanto, incluímos sua pesquisa em nossa árvore genealógica (Fig.3).

O projeto de pesquisa que está desenvolvendo atualmente tem como objetivo investigar questões relacionadas ao desenvolvimento profissional de professores de matemática em cenários de modelagem. Em Villarreal e Esteley (2013) abordam um dos objetivos específicos desse projeto, a saber, analisar o papel de diferentes mídias nesses cenários. A pesquisa foi desenvolvida de forma colaborativa entre as pesquisadoras e professoras do ensino médio, que discutiram sobre modelagem, trabalharam na modelagem de situações e planejaram (e aplicaram) uma proposta de modelagem para seus alunos do ensino médio.

Segundo Villarreal e Esteley (2013) o processo de modelagem desenvolvido pelos estudantes desenvolveu-se por um coletivo de atores humanos (docentes, estudantes e pesquisadores) e não humanos (instrumentos de medida, calculadoras, celulares). Além disso, as autoras apontam que o trabalho com tecnologias como a calculadora permitiu que um modelo matemático para a situação investigada fosse elaborado, mesmo os alunos tendo dificuldades em conhecimentos prévios. As autoras concluem que é possível aprender matemática em um ambiente de modelagem com tecnologias.

Com base nos trabalhos citados nesta seção, expandimos a árvore genealógica da Fig.2, acrescentando as pesquisas desenvolvidas e/ou orientadas pelos autores referenciados. A Fig.3 apresenta a expansão dessa árvore, apresentando o foco das novas pesquisas e indicando o tipo de orientação que está vinculada, quando pertinente. Nessa árvore genealógica, as linhas inclinadas do tipo traço, ponto, ponto, traço (-০০) indicam que a pesquisa foi desenvolvida 
juntamente com alguma orientação; já as linhas diagonais contínuas, indicam uma proposta de pesquisa em projeto trienal ou de doutoramento. Além disso, em cinza claro está indicada a pesquisa de doutorado de Villarreal (1999) que não tratou de modelagem. Esse é apenas um link visual para representar a ligação da autora com o grupo enquanto estudante. 


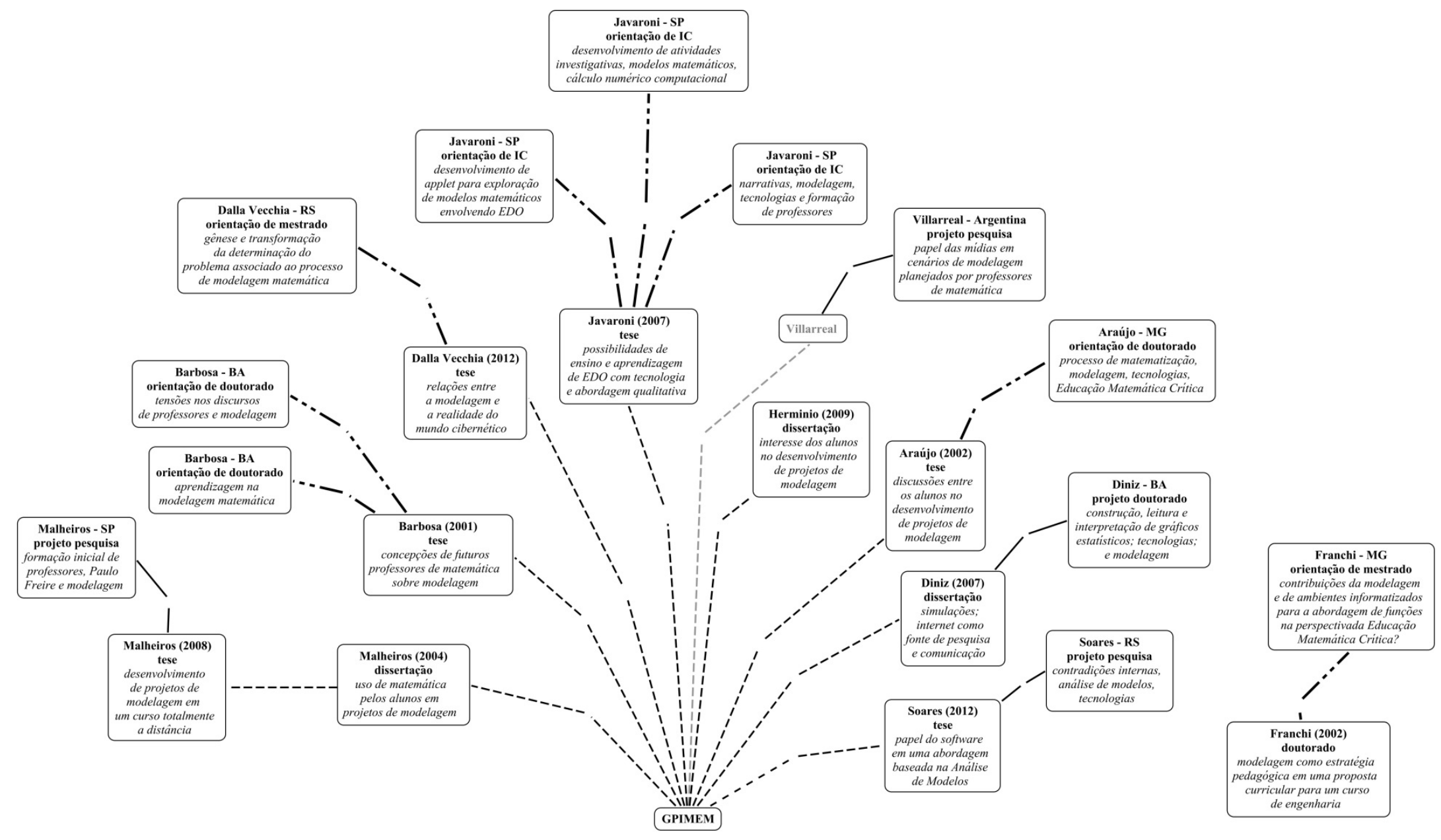

Figura 3: Árvore genealógica sobre as questões investigadas em pesquisas sobre modelagem desenvolvidas por seus autores enquanto alunos do GPIMEM e enquanto professores/pesquisadores/orientadores de universidades. 


\section{Discussão}

Em Borba (2000), Araujo e Borba (2004), Borba e Villarreal (2005), Maltempi, Javaroni e Borba (2011) e Chiari e Borba (2013) discutimos a dinâmica da pesquisa. Propusemos que é fundamental que - com as restrições de tempo impostas às investigações (prazos para mestrado, doutorado e projetos de docentes) - as pesquisas sejam desenvolvidas de forma coletiva. Assim a noção de mosaico de pesquisas surge para indicar que uma tese complemente uma iniciação científica, que por sua vez dialoga com o foco de um mestrado, e que são sintetizados em resultados de projetos coordenados por docentes ou doutorandos.

Indicamos também que, em nossa área de investigação, é mais comum ainda que haja ensino em diversas direções: um aluno de iniciação científica ensina algo a doutores, da mesma forma que pós-doutorandos ensinam a mestres. Não há um movimento da periferia ao centro, onde no centro se encontraria o coordenador do grupo, ou um núcleo duro.

\footnotetext{
Esta forma de encarar os novos desafios da sociedade moderna, amplamente tecnológica e informatizada, bem como os grandes projetos os quais desenvolveu, três deles mencionados anteriormente, levou o GPIMEM a desenvolver a postura do pesquisar em grupo, em um processo coletivo e colaborativo (CHIARI; BORBA, 2013).
}

Esses autores se referem a projetos de infraestrutura que ajudaram este grupo de pesquisa a ter espaço físico próprio e tecnologia digital que não dependesse apenas dos sempre constritos orçamentos das universidades públicas brasileiras. Outros aspectos da dinâmica da pesquisa em grupo também foram tratados nas referências acima citadas. Neste artigo, pretendemos analisar de forma distinta os caminhos de um grupo que não se propõe a "amarrar" em estrutura centralizada os mestrandos e doutorandos formados no grupo. Há diversos modelos de grupo de pesquisa. Há aqueles que se restringem aos membros que participam fisicamente de encontros regulares. Há outros, em que se tenta criar minigrupos de pesquisa semelhantes a um grupo central, ou mesmo aqueles que têm apenas uma estrutura centralizada, onde devido a questões geográficas, os encontros tem periodicidade esparsa.

O GPIMEM, desde sua conferência dos dez anos (já temos 21 anos no momento) refutou a ideia de criar GPIMEM em diversos estados ou países, e incentivou que seus membros egressos da UNESP, onde se baseia sua sede, criassem novos grupos, ou trabalhassem de 
forma individual. Há sim a possibilidade de que um membro tenha um vínculo mais solto, o de pesquisador associado, onde há um projeto, em geral menos ambicioso, e que permita que este pesquisador percorra caminhos distintos do GPIMEM. Tal modelo tem se mostrado interessante, na medida em que há uma constante oxigenação do grupo por meio do desenvolvimento de outros grupos e da sua influência no GPIMEM através de pesquisadores associados, ou de outros canais. Assim o GPIMEM parece reproduzir em nível de grupos a ideia, já consagrada em nível individual, que não há uma verticalidade, e sim uma horizontalidade, no melhor sentido Freireano, nas relações de ensino. Os grupos, novos e maduros, são educandos e educadores.

Desse modo, a ideia de construir uma genealogia de modelagem se encaixa neste mosaico de reflexões sobre a pesquisa realizada em grupos de pesquisa. O GPIMEM tem o foco em investigar o papel das tecnologias digitais em educação matemática, mas aceita, dentro da perspectiva de oxigenação aventada acima, teses como a de Barbosa (2001) que não tematizaram a informática. Modelagem tem sido a principal vertente pedagógica adotada nos trabalhos e investigações do grupo, dentro de uma visão de que as tecnologias digitais não são pedagogia; elas são parte de um coletivo formado por humanos e não humanos que constroem conhecimento. Entendemos que a Modelagem tem particular sinergia com as tecnologias digitais (BORBA, 2009; BORBA, 2012; SOARES; BORBA, 2014) porque elas podem ser das poucas alternativas pedagógicas a serem utilizadas caso a internet seja aceita em sala de aula. Em uma sala de aula onde a internet seja aceita, exercícios tradicionais já estarão resolvidos. Restará aos alunos construírem projetos de investigação e desenvolve-los em uma perspectiva da modelagem. Mas é claro que essas são conjecturas e nos interessa ver quais as avenidas que vem sendo desenvolvidas por pesquisadores mais ou menos vinculados ao GPIMEM depois de sua passagem pelo núcleo central do grupo. Nesse sentido é que nos esforçamos em construir essas árvores genealógicas.

A árvore genealógica da Fig.3 resume as questões sobre modelagem tematizadas pelas pesquisas desenvolvidas como mestrado e/ou doutorado no GPIMEM e também como orientação e/ou projeto de pesquisa de seus respectivos autores. Analisando mais especificamente os últimos trabalhos, é possível averiguar que a variedade de temas a respeito 
da modelagem permaneceu, sendo que, em alguns casos, as pesquisas seguiram assuntos relacionados àqueles pesquisados no mestrado e/ou doutorado, como é o caso dos trabalhos orientados por Javaroni e Dalla Vecchia, e a pesquisa proposta por Soares. Por outro lado, em outros casos as pesquisas tomaram rumos diferenciados daquelas do mestrado e/ou doutorado, como o projeto desenvolvido por Malheiros e as orientações de Araújo.

Outra observação significativa, é que nem todas as pesquisas continuaram a tratar ou a envolver modelagem e tecnologias. Por exemplo, a pesquisa de Malheiros (2013) relacionando modelagem e Paulo Freire não discute, pelo menos até o momento, questões envolvendo a tecnologia. Araújo e Diniz também possuem orientações cujos trabalhos versam sobre modelagem, mas não sobre tecnologias. No caso das orientações, essa mudança poderia estar diretamente relacionada ao interesse do próprio orientando, incentivado pelo professor a escolher um assunto de seu interesse como mote para desenvolvimento da pesquisa.

Já nas pesquisas envolvendo modelagem e tecnologias, podemos fazer um exercício semelhante àquele desenvolvido por Malheiros e Franchi (2013) e identificar qual o papel da tecnologia nesses estudos. Com base nos relatos que apresentamos na seção anterior, sugerimos alguns dos papéis possíveis e identificamos os trabalhos aos quais se referem: (i) contribuir para a compreensão de modelos matemáticos e dos fenômenos relacionados (SANTOS; JAVARONI, 2012); (ii) fornecer representações dos modelos matemáticos evidenciando aspectos das equações que não são facilmente observados apenas a partir de sua representação algébrica (SANTOS; JAVARONI, 2012; SGARIBOLDI; JAVARONI, 2013); (iii) fornecer possibilidades para a exploração, elaboração e/ou decisão sobre o modelo matemático a ser adotado (SGARIBOLDI, JAVARONI, 2013; FRANCHI; FERREIRA, 2013; FREITAS, 2011); (iv) "estopim” para o estudo de determinado conteúdo matemático, como os diferentes métodos numéricos utilizados pelos diferentes softwares (SGARIBOLDI; JAVARONI, 2013); (v) vetor de mudança, influenciando na determinação do problema a ser investigado e na maneira de representação de um problema (DALLA VECCHIA; MALTEMPI, 2012); (vi) contribuir para a exploração de situações reais e elaboração de modelos, mesmo com dificuldades em conhecimentos prévios (VILLARREAL; ESTELEY, 2013). 
Esses diferentes papéis da tecnologia que sugerimos testemunham, mais uma vez, a continuidade da evolução das pesquisas em termos desse tema, uma vez que papéis identificados em pesquisas recentes puderam ser revistos nos trabalhos analisados e, além disso, novos papéis puderam ser evidenciados. A tecnologia, desenvolvendo papel central nos processos de produção de conhecimento, atua nos coletivos seres-humanos-com-mídias (BORBA; VILLARREAL, 2005) que elaboram projetos de modelagem, reorganizando essa atividade a todo o momento. Novos papéis e potencialidades podem surgir conforme diferentes tecnologias forem utilizadas no fazer modelagem.

As pesquisas desenvolvidas por Villa-Ochoa ${ }^{5}$, por exemplo, têm potencial para agregar novos resultados ao mosaico em evolução. Esse pesquisador, proveniente da Colômbia, ingressou no GPIMEM ao fazer estágio "sanduíche" no grupo durante seu doutorado. Ele lidera em seu país uma rede virtual de professores que utilizam modelagem e atualmente desenvolve pesquisas sobre o tema, apoiado na noção de seres-humanos-com-mídias. Em Villa-Ochoa e Vahos (2010), por exemplo, os autores discutem sobre a interação de pesquisadores com o software GeoGebra e o surgimento de ideias para o planejamento de situações-problema que favoreçam o surgimento do pensamento variacional. Algumas dessas ideias baseiam-se em propriedades que, segundo os autores, não haviam sido percebidas em seus estudos anteriores com base em livros de matemática. Nesse caso, o contexto não é o desenvolvimento de projetos de modelagem, mas as conclusões dos autores já sugerem novos resultados envolvendo o construto seres-humanos-com-mídias.

Finalmente, com relação ao aspecto de disseminação do grupo, vemos que seus membros que desenvolveram pesquisas sobre modelagem em sua formação de mestres e/ou doutores estão concentrados principalmente na região Sudeste (estados de São Paulo e Minas Gerais), mas também estão presentes na região Sul (Rio Grande do Sul), na região Nordeste (Bahia) e no exterior (Argentina). Em particular, no caso do estado de São Paulo, as duas pesquisadoras (Malheiros e Javaroni) continuam diretamente vinculadas ao GPIMEM, orientando trabalhos de mestrado em diferentes temas.

\footnotetext{
${ }^{5} \mathrm{O}$ trabalho de Villa-Ochoa não foi incluído nas árvores desenvolvidas nesse artigo, mas certamente estará presente em uma ampliação das mesmas.
} 
Com base nessas informações, podemos visualizar e identificar os diferentes caminhos seguidos pela modelagem enquanto foco de pesquisas desenvolvidas e/ou orientadas por exalunos do GPIMEM. É claro que esse levantamento é parcial e, de fato, não temos a intenção de ser exaustivos. Porém, os aspectos mencionados acima ilustram a dinamicidade e a vida do GPIMEM em termos de modelagem que, direta ou indiretamente, é mantida pelo trabalho daqueles que um dia foram seus alunos e hoje são professores, pesquisadores e/ou orientadores de diferentes universidades dentro e fora do país.

\section{Referências}

ANDRADE, R. F. S. Modelagem Matemática: uma experiência na educação de jovens e adultos. 2010. Monografia (Licenciatura em Matemática) - Centro de Formação de Professores, Universidade Federal do Recôncavo da Bahia, Amargosa, 2010.

ARAÚJO, J. L. Cálculo, Tecnologias e Modelagem Matemática: as discussões dos alunos. 2002. 173f. Tese (Doutorado em Educação Matemática) - Instituto de Geociências e Ciências Exatas, Universidade Estadual Paulista, Rio Claro, 2002.

ARAÚJO, J. L.; BORBA, M. C. Construindo Pesquisas Coletivamente em Educação Matemática. In: BORBA, M. C.; ARAÚJO, J. L. (Org.) Pesquisa Qualitativa em Educação Matemática. Belo Horizonte: Autêntica, 2004. p.25-45.

BARBOSA, J. C. Modelagem Matemática: concepções e experiências de futuros professores. 2001. 253f. Tese (Doutorado em Educação Matemática) - Instituto de Geociências e Ciências Exatas, Universidade Estadual Paulista, Rio Claro, 2001.

BORBA, M. C. GPIMEM e UNESP: Pesquisa, Extensão e Ensino em Informática e Educação Matemática. In: PENTEADO, M. G.; BORBA, M. C. (Org.) A Informática em Ação: formação de professores, pesquisa e extensão. Rio Claro: Olhos d'Água, 2000. p.47-66.

BORBA, M. C. Potential scenarios for Internet use in the mathematics classroom. International Journal on Mathematics Education (ZDM), v.41, p.453-465, 2009.

BORBA, M. C. Humans-with-media and continuing education for mathematics teachers in online environments. International Journal on Mathematics Education (ZDM), v.44, p.802-814, 2012.

BORBA, M. C.; PENTEADO, M. G. Informática e Educação Matemática. (Coleção Tendências em Educação Matemática). Belo Horizonte: Autêntica, 2001. 
BORBA, M. C.; SKOVSMOSE, O. A ideologia da certeza em Educação Matemática. In: SKOVSMOSE, O. Educação matemática crítica: a questão da democracia. Campinas: Papirus, 2001. p.127-148.

BORBA, M. C.; VILLARREAL, M. E. Humans-with-Media and the Reorganization of Mathematical Thinking: information and communication technologies, modeling, experimentation and visualization. New York: Springer, 2005.

COELHO, K. R. ; JAVARONI, S. L. ; BARALDI, I. M. ; FINATO, J. A. R. Cheiros, sabores e memórias de professores de matemática: uma experiência saudável com modelagem matemática. In: Congresso de Iniciação Científica da Unesp, 22 (XXII CIC). 2010. Bauru, SP. Anais... Bauru: Universidade Estadual Paulista, 2010. p.1-4. Disponível em: $<$ http://prope.unesp.br/cic_isbn/busca.php> Acesso em: 06 Mar. 2014.

CHIARI, A. S. S.; BORBA, M. C. Vinte anos de GPIMEM: um mosaico de pesquisas em movimento. In: BORBA, M. C.; CHIARI, A. S. S. (Org.) Tecnologias Digitais e Educação Matemática. $1^{\mathrm{a}}$ Ed. São Paulo: Editora Livraria da Física, 2013. p.13-37.

DALlA VECCHIA, R. A Modelagem Matemática e a Realidade do Mundo Cibernético. 2012. 275f. Tese (Doutorado Educação Matemática) - Instituto de Geociências e Ciências Exatas, Universidade Estadual Paulista, Rio Claro, 2012.

DALLA VECCHIA, R.; MALTEMPI, M. V. Modelagem Matemática e Tecnologias de Informação e Comunicação: a realidade do mundo cibernéticos como um vetor de virtualização. Boletim de Educação Matemática (BOLEMA), v.26, n.43, p.191-218, 2012.

DELEUZE, G. Diferença e Repetição. Traduzido por: Luiz Orlandi; Roberto Machado. Tradução de: Différence et Répétition. Rio de Janeiro: Graal, 1988.

DINIZ, L. N. O papel das tecnologias da informação e comunicação nos projetos de modelagem. 2007. 118f. Dissertação (Mestrado em Educação Matemática) - Instituto de Geociências e Ciências Exatas, Universidade Estadual Paulista, Rio Claro, 2007.

DINIZ, L. N.; BORBA, M. C. Leitura e Interpretação de "Dados Prontos" em um Ambiente de Modelagem e Tecnologias Digitais: o mosaico em Movimento. Boletim de Educação Matemática (Bolema), v.26, n.43, 2012. p.163-190.

DINIZ, L. N.; SOUZA, N. F. Reflexões sobre os Aspectos a Considerar na Organização de Atividades Envolvendo a Interpretação de Gráficos Estatísticos. In: Encontro de Probabilidades e Estatística na Escola, 3., 2013, Braga, Portugal. Atas... Braga: [s.i.], 2013. Disponível em: https://repositorium.sdum.uminho.pt/bitstream/1822/23169/1/2013AtasIIIEPEE.pdf >. Acesso em 15 mar. 2014. 
ENGESTRÖM, Y.; SANNINO, A. Studies of Expansive Learning: foundations, findings and future challenges. Educational Research Review, v.5, p.1-24, 2010.

FRANCHI, R. H. O. L. Uma proposta curricular de Matemática para cursos de Engenharia utilizando Modelagem Matemática e Informática. 2002. Tese (Doutorado em Educação Matemática) - Instituto de Geociências e Ciências Exatas, Universidade Estadual Paulista, Rio Claro, 2002.

FRANCHI, R. H. O. L.; FERREIRA, N. S. The potential of Mathematical Modelling activities for critical reflection and the teaching of Mathematics. In: International Conference on the Teaching of Mathematical Modelling and Applications, 16 (16 ${ }^{\text {th }}$ ICTMA). 2013. Blumenau, SC. ICTMA 16 Abstracts, 2013. p.88.

FREITAS, W. S. A modelagem na perspectiva da Educação Matemática Crítica. In: Conferência Nacional sobre Modelagem na Educação Matemática, 7 (VII CNMEM). 2011. Belém, PA. Anais... Pará: Universidade Federal do Pará, 2011. p.1-22. CD-ROM.

HERMINIO, M. H. G. B. O processo de escolha dos temas dos projetos de modelagem. 2009. 139f. Dissertação (Mestrado em Educação Matemática) - Instituto de Geociências e Ciências Exatas, Universidade Estadual Paulista, Rio Claro, 2009.

HOUAISS, A. Dicionário Houaiss da Língua Portuguesa. Rio de Janeiro: Editora Objetiva, 2001.

JAVARONI, S. L. Abordagem geométrica: possibilidades para o ensino e aprendizagem de Introdução às Equações Diferenciais Ordinárias. 2007. 231f. Tese (Doutorado Educação Matemática) - Instituto de Geociências e Ciências Exatas, Universidade Estadual Paulista, Rio Claro, 2007.

MALHEIROS, A. P. S. A produção matemática dos alunos em um ambiente de modelagem. 2004. 180f. Dissertação (Mestrado em Educação Matemática) - Instituto de Geociências e Ciências Exatas, Universidade Estadual Paulista, Rio Claro, 2004.

MALHEIROS, A. P. S. Educação Matemática online: a elaboração de projetos de Modelagem. 2008. Tese (Doutorado Educação Matemática) - Instituto de Geociências e Ciências Exatas, Universidade Estadual Paulista, Rio Claro, 2008.

MALHEIROS, A. P. Diálogos entre Modelagem e Paulo Freire: possibilidades para a sala de aula. In: Conferência Nacional sobre Modelagem na Educação Matemática, 8 (VIII CNMEM). 2013. Santa Maria, RS. Anais... Santa Maria: Centro Universitário Franciscano, 2013. V. único. p.1-10. CD-ROM.

MALHEIROS, A. P. S.; FRANCHI, R. H. O. L. As Tecnologias da Informação e Comunicação nas produções sobre Modelagem no GPIMEM. In: BORBA, M. C.; CHIARI, A. 
(Orgs.) Tecnologias Digitais e Educação Matemática, São Paulo: Editora Livraria da Física, 2013. p.175-193.

MALTEMPI, M. V.; JAVARONI, S. L.; BORBA, M. C. Calculadoras, Computadores e Internet em Educação Matemática - dezoito anos de pesquisa. Boletim de Educação Matemática (BOLEMA), v.25, p.43-72, 2011.

OLIVEIRA, A. M. P. Modelagem Matemática e as tensões nos discursos dos professores. 2010. 199f. Tese (Doutorado em Ensino, Filosofia e História das Ciências) - Instituto de Física, Universidade Federal da Bahia. Universidade Estadual de Feira de Santana, 2010.

SANTOS, M. C. M. ; JAVARONI, S. L. Modelagem Matemática e o Desenvolvimento de Applets. In: Congresso de Iniciação Científica da Unesp, 24 (XXIV CIC). 2012. Bauru, SP. Anais... Bauru: Universidade Estadual Paulista, 2012. p.1-1. Disponível em: $<$ http://prope.unesp.br/cic_isbn/busca.php> Acesso em: 06 Mar. 2014.

SGARIBOLDI, M. L.; JAVARONI, S. L. Cálculo Numérico na Engenharia: potencialidades do uso de modelagem matemática auxiliada pelas tecnologias informáticas. In: Congresso de Iniciação Científica da Unesp, 25 (XXV CIC). 2013. Bauru, SP. Anais... Bauru: Universidade Estadual Paulista, 2013. p.1-1. Disponível em: <http://prope.unesp.br/cic_isbn/busca.php $>$ Acesso em: 06 Mar. 2014.

SILVA, M. S.; BARBOSA, J. C.; OLIVEIRA, A. M. P. Materiais Curriculares Educativos sobre Modelagem Matemática e a recontextualização pedagógica operada por professores iniciantes. Unión (San Cristoban de La Laguna), v.34, p.47-67, 2013.

SOARES, D. S. Uma Abordagem Pedagógica Baseada na Análise de Modelos para Alunos de Biologia: qual o papel do software? 2012. 341f. Tese (Doutorado Educação Matemática) - Instituto de Geociências e Ciências Exatas, Universidade Estadual Paulista, Rio Claro, 2012.

SOARES, D. S.; BORBA, M. C. The role of software Modellus in a teaching approach based on model analysis. The International Journal on Mathematics Education, 2014, DOI 10.1007/s11858-013-0568-5. Disponível em: $<$ http://link.springer.com/article/10.1007/s11858-013-0568-5 > Acesso em: 17 mar. 2014.

SOUZA, A. G. O. A Modelagem Matemática Proporcionando Significado aos Conteúdos Matemáticos. 2013. Monografia (Licenciatura em Matemática) - Centro de Formação de Professores, Universidade Federal do Recôncavo da Bahia, Cruz das Almas, 2013.

SOUZA, E. G. A aprendizagem matemática na modelagem matemática. 2012. Tese (Doutorado em Ensino, Filosofia e História das Ciências) - Instituto de Física, Universidade Federal da Bahia. Universidade Estadual de Feira de Santana, 2012. 
VILLA-OCHOA, J. A.; VAHOS, M. R. Pensamiento variacional: seres-humanos-conGeoGebra en la visualización de nociones varacionales. Educação Matemática Pesquisa, v.12, n.3, p.514-528, 2010.

VILLARREAL, M. E. O pensamento matemático de estudantes universitários de Cálculo e tecnologias informáticas. 1999. 389f. Tese (Doutorado Educação Matemática) - Instituto de Geociências e Ciências Exatas, Universidade Estadual Paulista, Rio Claro, 1999.

VILLARREAL, M. E.; ESTELEY, C. B. Escenarios de Modelización y Medios: acciones, actividades y diálogos. In: BORBA, M. C.; CHIARI, A. (Orgs.) Tecnologias Digitais e Educação Matemática, São Paulo: Editora Livraria da Física, 2013. p.273-308. 